\title{
Treatment progression in sulfonylurea and dipeptidyl peptidase- 4 inhibitor cohorts of type 2 diabetes patients on metformin
}

This article was published in the following Dove Press journal:

Patient Preference and Adherence

10 August 2016

Number of times this article has been viewed

\section{Xiaomei Peng \\ Dingfeng Jiang \\ Dongju Liu \\ Oralee J Varnado \\ Jay $\mathrm{P}$ Bae}

Eli Lilly and Company, Global Patient Outcomes and Real World Evidence, Indianapolis, IN, USA
Correspondence: Xiaomei Peng

Eli Lilly and Company, Corporate Center, Indianapolis, IN, 46285, USA

Tel + I 3 I7 4339534

Email peng_xiaomei@lilly.com
Background: Metformin is an oral antidiabetic drug (OAD) widely used as first-line therapy in type 2 diabetes (T2D) treatments. Numerous treatment pathways after metformin failure exist. It is important to understand how treatment choices influence subsequent therapy progressions. This retrospective study compares adherence to, persistence with, and treatment progression in sulfonylurea (SU) and dipeptidyl peptidase-4 (DPP-4) inhibitor patient cohorts with T2D on metformin.

Methods: Using health insurance claims data, matched patient cohorts were created and OAD use was compared in patients with T2D initiating SU or DPP-4 inhibitors (index drugs) since January 1, 2010, to December 31, 2010, with background metformin therapy. Propensity score matching adjusted for possible selection bias. Persistence was measured via Cox regression as days to a $\geq 60$-day gap in index drug possession; adherence was defined as proportion of days covered (PDC) $\geq 80 \%$. Evolving treatment patterns were traced at 6 -month intervals for 24 months following index drug discontinuation.

Results: From among 19,621 and 7,484 patients in the SU and DPP-4 inhibitor cohorts, respectively, 6,758 patient pairs were matched. Persistence at 12 months in the SU cohort was $48.0 \%$ compared to $52.5 \%$ for the DPP-4 inhibitor cohort. PDC adherence (mean [SD]) during the 12-month follow-up period was 63.3 (29.7) for the SU cohort and 65.5 (28.7) for the DPP-4 inhibitor cohort. PDC $\geq 80 \%$ was $40.5 \%$ and $43.4 \%$ in the SU and DPP-4 inhibitor cohorts, respectively. A higher percentage of patients in the SU cohort remained untreated. Following index drug discontinuation, monotherapy was more common in the SU cohort, while use of two or three OADs was more common in the DPP-4 inhibitor cohort. Insulin therapy initiation was higher in the SU cohort.

Conclusion: Slightly better adherence and persistence were seen in the DPP-4 inhibitor cohort. Adherence and persistence remain a challenge to many patients; understanding therapy progression will help identify target areas for intervention and improvement.

Keywords: adherence, sulfonylurea, DPP-4 inhibitor, metformin

\section{Introduction}

Type 2 diabetes (T2D) is a progressive metabolic disease characterized by insulin resistance and pancreatic beta-cell dysfunction. The American Diabetes Association and European Association for the Study of Diabetes recommend initial treatment regimens consisting of diet and exercise, weight control, increased physical activity, smoking cessation, and lipid management, followed by metformin administration. ${ }^{1,2}$

Metformin is an oral antidiabetic drug (OAD), and it is the most widely used firstline therapy in T2D treatments. ${ }^{3,4}$ The progressive nature of T2D warrants the use of 
other agents, such as sulfonylureas (SUs), thiazolidinediones, dipeptidyl peptidase-4 (DPP-4) inhibitors, glucagon-like peptide-1 receptor agonists, sodium-glucose cotransporter-2 inhibitors, or insulin, either as monotherapy or in addition to metformin. . $^{1,2}$

Adherence to and persistence with OAD agents such as SUs and DPP-4 inhibitors are often suboptimal, and discontinuation rates are high. Studies document that treatment adherence in patients with T2D is often poor due to complicated treatment regimens, medication costs, and tolerability issues; better adherence is often associated with fewer daily doses of medications. ${ }^{5-9}$

Furthermore, in a retrospective analysis comparing adherence and persistence in patients treated with SUs or DPP-4 inhibitors, $41 \%$ of SU-treated and $47 \%$ of DPP-4 inhibitortreated patients were adherent during the 1 -year follow-up. ${ }^{10}$ A greater percentage of patients in the SU cohort $(52 \%)$ discontinued treatment in comparison to the DPP-4 inhibitor cohort (45\%). ${ }^{10}$ Similar results were observed for the 2-year follow-up. ${ }^{10}$ The results of Rathmann et al ${ }^{11}$ were comparable to these results considering that $49 \%$ of SU-treated and $39 \%$ of DPP-4 inhibitor-treated patients had discontinued treatment by the 24-month follow-up. As there are many possible treatment pathways for T2D given the array of drugs available, it is important to understand use patterns not only in terms of combination therapies but also with respect to different treatment pathways.

In this study, we attempt to compare adherence to and persistence with SUs and DPP-4 inhibitors as an augmentation after metformin monotherapy. In addition, this study examines how patients progress through treatment pathways after discontinuing second-line OAD by following treatment dispositions for 24 months thereafter. With the continuing follow-up (or observation) of patients' treatment regimens after discontinuation of the index OAD for up to 2 years, this study describes the progression of therapy, including alternative OAD regimens and injectable therapies. Treatment patterns observed from this study may be helpful for clinicians when considering treatment options for patients with T2D.

\section{Methods}

This retrospective observational study utilized insurance claims data to compare patients who were prescribed SUs or DPP-4 inhibitors. Data for this analysis were collected from the Truven Health Analytics MarketScan ${ }^{\circledR}$ Commercial Claims and Encounters database for the period 2009-2013 and the Medicare Supplemental and Coordination of Benefits database. These databases represent the health services of $\sim 195$ million employees, dependents, and retirees in the USA with primary or Medicare supplemental coverage through privately insured fee-for-service, point-of-service, or capitated health plans. The data collected from the databases are generally representative of the treated population in the USA. All enrollment records and drug claims were collected for the SU and DPP-4 inhibitor cohorts described herein.

For each cohort (Figure 1), eligible patients were those who initiated at least one prescription fill of the index drug (SU or the two DPP-4 inhibitors saxagliptin and sitagliptin, but not both index drugs) since January 1, 2010, to December 31, 2010 (index event period) with background metformin therapy; had $\geq 1$ diagnoses of $T 2 D$; were $\geq 18$ years old at the index date (date on which index drug was initiated) and had 24 months (12 months prior to and 12 months after index date) continuous pharmaceutical and medical benefit enrollment; and had $\geq 30$ days of total supply for the index drug. Background metformin therapy was defined as evidence of possession of metformin prescription at the start or during the index period and also having at least one metformin claim within 6 months after index end date. Eligible patients did not have a prescription claim for the index drug for the previous 90 days in each respective cohort, in addition to not having type 1 diabetes, secondary diabetes, or gestational conditions during the baseline period (12 months prior to the index date).

The outcome measures were persistence, as measured by days to discontinuation of index drug, and adherence, as measured by proportion of days covered (PDC). Patients were considered to have discontinued treatment if the gap between the end date of a prior index drug claim and start date of a current index drug claim was $\geq 60$ days. Patients who had not discontinued at the last day of postindex follow-up (12 months, counted as 365 days after the index start date) were censored at this time point. PDC was defined as the proportion of days that the patient had the index drug supply on hand during the 365-day follow-up period. The PDC provides a composite measure of both adherence prior to discontinuation and persistence during the entire follow-up period. It is generally accepted that patients with PDC of $\geq 80 \%$ are adherent. ${ }^{12}$ This is retrospective database study. The database has been de-identified and anonymized data that is HIPAA compliant was used; therefore, patient consent and ethical approval were not obtained.

\section{Statistical analysis}

Propensity score matching was used to control for possible selection bias. Logistic regression was used to estimate the propensity score with the baseline variables capturing demographics, comorbidities and disease burden, comedication, 


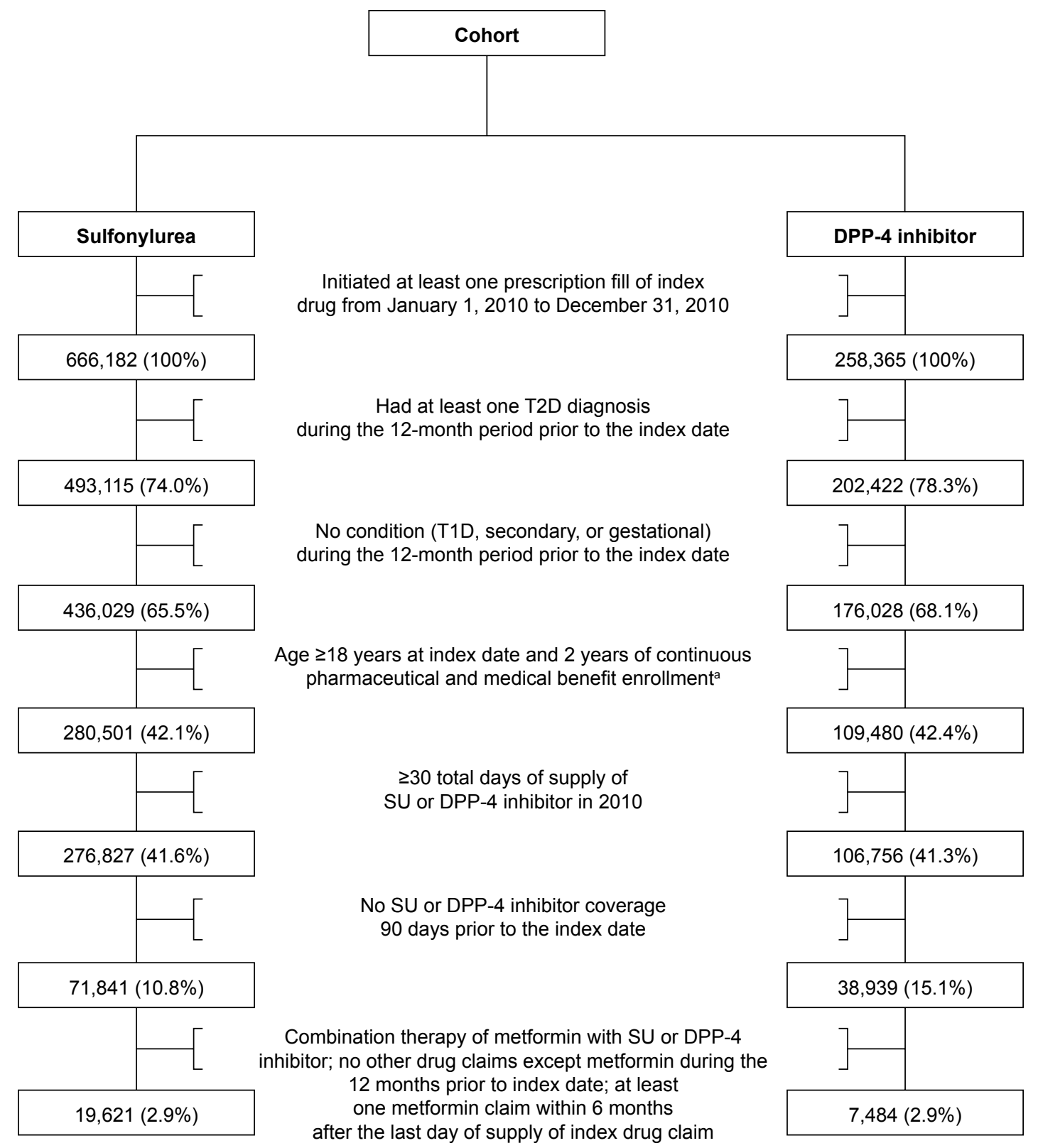

Figure I Flow diagram for patient selection.

Note: a'Two years' continuous enrollment includes I year prior to and I year postindex date.

Abbreviations: DPP-4, dipeptidyl peptidase-4; SU, sulfonylurea; TID, type I diabetes; T2D, type 2 diabetes.

region, insurance plan, and economic status. A greedy 1:1 matching algorithm ${ }^{13}$ was utilized to match each SU-treated patient with a similar DPP-4 inhibitor-treated patient.

Baseline characteristics of the SU and DPP-4 inhibitor cohorts were summarized and compared using the Wilcoxon rank sum test for continuous variables and chi-square test for categorical variables. Kaplan-Meier estimates and Cox regression were used to describe and compare, respectively, the persistence of patients in each cohort. $T$-tests were used to analyze the PDC, and the chi-square test was used to analyze adherence defined as achieving $\geq 80 \%$ PDC.

\section{Results}

Prior to executing propensity score matching, there were 19,621 and 7,484 patients in the SU and DPP-4 inhibitor cohorts, respectively (Figure 1). Table 1 provides the baseline characteristics before and after propensity score matching. A majority of the baseline variables showed a significant $(P<0.05)$ difference between the cohorts before matching, and showed no significant difference after matching. Although the number of antidiabetic drug classes and unique antidiabetic generic drug names remained significant after matching, the mean and standard deviation (SD) of both 
Table I Pre- and postmatched baseline characteristics and demographics

\begin{tabular}{|c|c|c|c|c|c|c|}
\hline \multirow[t]{2}{*}{ Variable $^{a}$} & \multicolumn{3}{|l|}{ Prematched } & \multicolumn{3}{|l|}{ Postmatched } \\
\hline & $\begin{array}{l}\text { Sulfonylurea } \\
(N=19,621)\end{array}$ & $\begin{array}{l}\text { DPP-4 inhibitor } \\
(N=7,484)\end{array}$ & $P$-value & $\begin{array}{l}\text { Sulfonylurea } \\
(N=6,758)\end{array}$ & $\begin{array}{l}\text { DPP-4 inhibitor } \\
(\mathrm{N}=6,758)\end{array}$ & $P$-value \\
\hline \multicolumn{7}{|l|}{ Demographics } \\
\hline Age, years & $56.6(12.1)$ & $54.8(I I . I)$ & $<0.001$ & $54.3(11.4)$ & $54.6(11.1)$ & 0.064 \\
\hline Females & $8,492(43.3)$ & $3,507(46.9)$ & $<0.001$ & $3,121(46.2)$ & $3,113(46.1)$ & 0.890 \\
\hline \multicolumn{7}{|l|}{ Region } \\
\hline North-Central & $5,100(26.0)$ & $1,680(22.5)$ & $<0.00$ I & $1,588(23.5)$ & I,545 (22.9) & 0.664 \\
\hline Northeast & $|, 85|(9.4)$ & $\mathrm{I}, 007(13.5)$ & & $905(13.4)$ & $899(13.3)$ & \\
\hline South & $8,522(43.4)$ & $3,849(51.4)$ & & $3,447(51.0)$ & $3,444(51.0)$ & \\
\hline West & $4,120(21.0)$ & $940(12.6)$ & & $812(12.0)$ & $862(12.8)$ & \\
\hline Unknown & $28(0.1)$ & $8(0.1)$ & & $6(0.1)$ & $8(0.1)$ & \\
\hline \multicolumn{7}{|l|}{ Comorbidities/complications } \\
\hline Neurological symptoms & $2,547(13.0)$ & $879(11.8)$ & 0.006 & 805 (11.9) & 789 (II.7) & 0.670 \\
\hline Peripheral vascular disease & $\mathrm{I}, 40 \mathrm{I}(7 . \mathrm{I})$ & $497(6.6)$ & 0.150 & 401 (5.9) & $444(6.6)$ & 0.127 \\
\hline Cardiovascular disease & $12,315(62.8)$ & $4,667(62.4)$ & 0.538 & $4,163(61.6)$ & $4,21 I(62.3)$ & 0.395 \\
\hline Renal complications & $2,914(\mid 4.9)$ & $\mathrm{I}, 12 \mathrm{I}(15.0)$ & 0.793 & $\mathrm{I}, 020(\mid 5 . \mathrm{I})$ & $\mathrm{I}, 010(15.0)$ & 0.810 \\
\hline Endocrine/metabolic complications & $9,459(48.2)$ & $3,891(52.0)$ & $<0.00$ I & $3,526(52.2)$ & $3,501(51.8)$ & 0.667 \\
\hline Ophthalmic complications & $3,926(20.0)$ & $1,429(19.1)$ & 0.091 & $\mathrm{I}, 23 \mathrm{I}(\mathbf{1 8 . 2 )}$ & $1,256(18.6)$ & 0.579 \\
\hline Other complications & $3,143(16.0)$ & $\mathrm{I}, 022(13.7)$ & $<0.001$ & $931(13.8)$ & $933(13.8)$ & 0.960 \\
\hline Depression & $\mathrm{I}, 048(5.3)$ & $362(4.8)$ & 0.095 & $336(5.0)$ & $327(4.8)$ & 0.720 \\
\hline Obesity & I,382 (7.0) & $462(6.2)$ & 0.011 & $395(5.8)$ & $429(6.5)$ & 0.222 \\
\hline Osteoarthritis & I,886 (9.6) & $760(10.2)$ & 0.178 & $648(9.6)$ & $665(9.8)$ & 0.622 \\
\hline \multicolumn{7}{|l|}{ Medications } \\
\hline Number of antidiabetic drug classes & $1.2(0.7)$ & $1.0(0.6)$ & $<0.001$ & $0.9(0.7)$ & $0.9(0.6)$ & $<0.001$ \\
\hline Number of any medications & $8.7(5.5)$ & $8.9(5.6)$ & 0.098 & $8.6(6.0)$ & $8.6(5.4)$ & 0.059 \\
\hline Number of antidiabetic medications & I.I (0.7) & $0.9(0.5)$ & $<0.001$ & $0.8(0.6)$ & $0.9(0.5)$ & $<0.001$ \\
\hline \multicolumn{7}{|l|}{ Other } \\
\hline \multicolumn{7}{|l|}{ Plan type } \\
\hline FFS & $4,157(21.2)$ & I,397 (18.7) & $<0.001$ & $1,198(17.7)$ & $\mathrm{I}, 232(18.2)$ & 0.167 \\
\hline $\mathrm{HMO}$ & $3,945(20.1)$ & $933(12.5)$ & & $809(12.0)$ & $868(12.8)$ & \\
\hline PPO & II,050 (56.3) & $4,962(66.3)$ & & $4,558(67.5)$ & $4,492(66.5)$ & \\
\hline Missing & $469(2.4)$ & $192(2.6)$ & & $193(2.9)$ & $166(2.5)$ & \\
\hline $\mathrm{CCl}$ score & $1.6(1.2)$ & $\mathrm{I} .6(\mathrm{I} . \mathrm{I})$ & $<0.001$ & I.5 (I.I) & I.6 (I.I) & 0.243 \\
\hline
\end{tabular}

Note: a Data presented as $\mathrm{n}(\%)$ for categorical variables and mean (SD) for numeric variables.

Abbreviations: CCl, Charlson Comorbidity Index; DPP-4, dipeptidyl peptidase-4; FFS, fee for service; HMO, health maintenance organization; PPO, preferred provider organization.

variables were close. This difference was probably driven by the large sample size and does not confer clinical significance. Standardized differences ${ }^{13}$ in percentages were also calculated for these two variables, which indicated a good match for the number of antidiabetic drug classes $(8.1 \%)$ and the number of antidiabetic medications (9.8\%). The final number of patients in each matched propensity cohort was 6,758 (Table 1).

Figure $2 \mathrm{~A}$ and $\mathrm{B}$ shows the persistence rates of the $\mathrm{SU}$ and DPP-4 inhibitor cohorts before and after propensity score matching, respectively. Prior to propensity matching, $46.2 \%$ $(9,064$ of 19,621$)$ and $52.1 \%(3,900$ of 7,484$)$ of SU- and DPP-4 inhibitor-treated patients, respectively, remained persistent during the 12-month follow-up period (Figure 2A). The hazard ratio of discontinuation for DPP-4 inhibitors versus SU before matching was $0.846(P<0.001)$ (Figure 2A), ie, the DPP-4 inhibitor cohort was $\sim 15.4 \%$ less likely to discontinue the index drug. Following propensity matching,
$48.0 \%(3,241$ of 6,758$)$ and $52.5 \%(3,550$ of 6,758$)$ of SUand DPP-4 inhibitor-treated patients, respectively, remained persistent during the 12-month follow-up period (Figure 2B and Table 2). The hazard ratio of discontinuation for DPP-4 inhibitors versus SU after matching was $0.877(P<0.001)$ (Figure 2B), ie, the DPP-4 inhibitor cohort was $\sim 12.3 \%$ less likely to discontinue the index drug.

A summary of postmatching adherence as measured by PDC during the 12-month follow-up period is provided in Table 2. The PDC results (mean [SD]) showed slightly better adherence for the DPP-4 inhibitor cohort (65.5 [28.7]) in comparison to the SU cohort (63.3 [29.7]). This difference was statistically significant when evaluated using a twosample $t$-test $(P<0.001)$. The PDC of OAD and overall antidiabetic medication were similar in both cohorts. A higher percentage of patients in the DPP-4 inhibitor cohort (43.4\% $[2,930]$ ) met the PDC threshold of $\geq 80 \%$ (good adherence) 


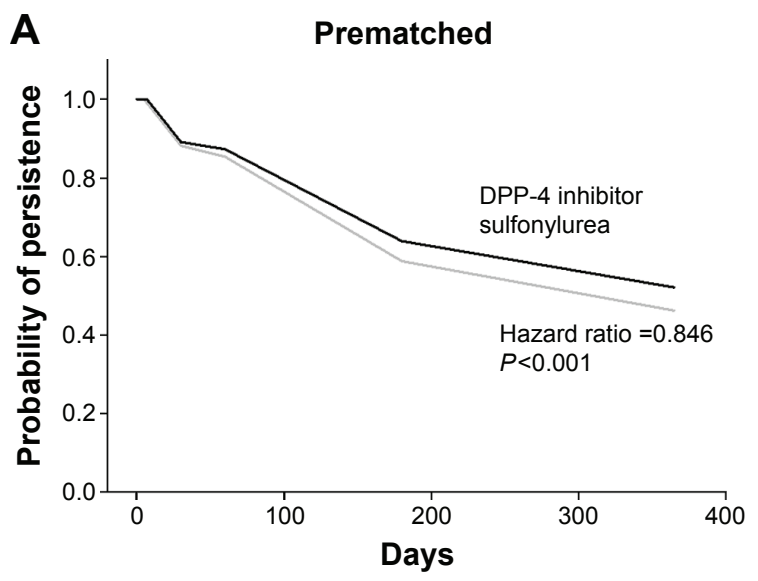

DPP-4 inhibitor

\begin{tabular}{|l|c|l|c|}
\hline Day & $\mathbf{n}(\%)^{\mathbf{a}}$ & Day & $\mathbf{n}(\%)^{\mathbf{a}}$ \\
\hline 0 & $7,484(100)$ & 0 & $19,621(100)$ \\
\hline 7 & $7,483(99.9)$ & 5 & $19,619(99.9)$ \\
\hline 30 & $6,671(89.1)$ & 30 & $17,307(88.2)$ \\
\hline 60 & $6,533(87.3)$ & 60 & $16,754(85.4)$ \\
\hline 180 & $4,784(63.9)$ & 180 & $11,541(58.8)$ \\
\hline 365 & $3,900(52.1)$ & 365 & $9,064(46.2)$ \\
\hline
\end{tabular}

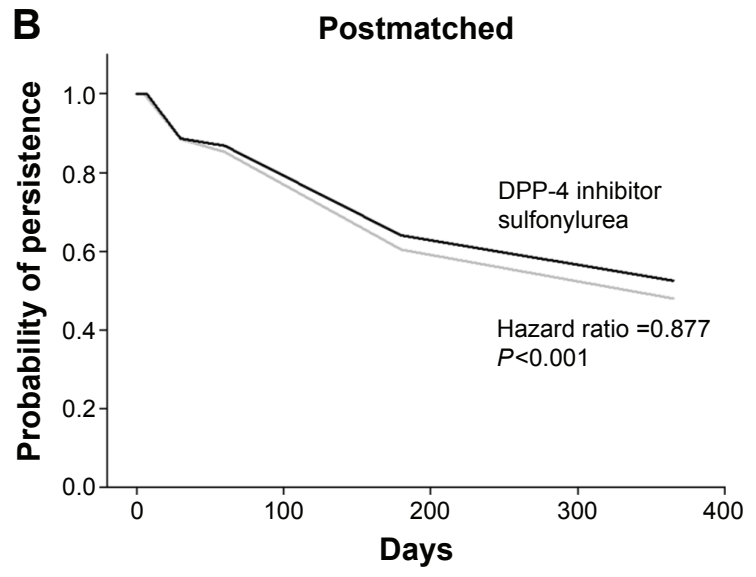

\begin{tabular}{|l|c|l|c|}
\hline \multicolumn{2}{|c|}{ DPP-4 inhibitor } & \multicolumn{1}{l|}{ SU } \\
\hline Day & $\mathbf{n}(\%)^{\mathrm{a}}$ & Day & $\mathbf{n}(\%)^{\mathrm{a}}$ \\
\hline 0 & $6,758(100)$ & 0 & $6,758(100)$ \\
\hline 7 & $6,757(99.9)$ & 5 & $6,757(99.9)$ \\
\hline 30 & $5,990(88.6)$ & 30 & $5,977(88.4)$ \\
\hline 60 & $5,865(86.8)$ & 60 & $5,760(85.2)$ \\
\hline 180 & $4,327(64.0)$ & 180 & $4,083(60.4)$ \\
\hline 365 & $3,550(52.5)$ & 365 & $3,241(48.0)$ \\
\hline
\end{tabular}

Figure 2 Pre- and postmatched persistence rates for SU versus DPP-4 inhibitors. Notes: Figure shows the persistence rate of the SU and DPP-4 inhibitor cohorts (A) before and (B) after propensity score matching. ${ }^{2}$ Represents the number of patients (\%) who continued the index drug.

Abbreviations: DPP-4, dipeptidyl peptidase-4; SU, sulfonylurea.

Table 2 Postmatched adherence and persistence during the I2-month follow-up

\begin{tabular}{|c|c|c|c|}
\hline & $\begin{array}{l}\text { Sulfonylurea } \\
(\mathrm{N}=6,758)\end{array}$ & $\begin{array}{l}\text { DPP-4 inhibitor } \\
(\mathbf{N}=6,758)\end{array}$ & $P$-value ${ }^{a}$ \\
\hline \multicolumn{4}{|l|}{ Persistence, n (\%) ${ }^{\mathrm{b}}$} \\
\hline Index drug & $3,24 \mid(48.0)$ & $3,550(52.5)$ & $<0.001$ \\
\hline \multicolumn{4}{|l|}{ PDC, ${ }^{\mathrm{c}}$ mean (SD) } \\
\hline SU class & $63.3(29.7)$ & $3.9(13.5)$ & $<0.001$ \\
\hline DPP-4 inhibitor class & $2.3(10.2)$ & $65.5(28.7)$ & $<0.001$ \\
\hline Antidiabetic drug (any) & $77.5(24.0)$ & $77.1(23.3)$ & 0.268 \\
\hline $\begin{array}{l}\text { Oral antidiabetic drug } \\
\text { (any) }\end{array}$ & $77.1(24.3)$ & $76.5(23.6)$ & 0.188 \\
\hline $\begin{array}{l}\text { Noninsulin antidiabetic } \\
\text { injectables }\end{array}$ & $0.5(4.4)$ & $0.9(5.8)$ & $<0.001$ \\
\hline Insulin (any) & I.I (6.4) & $0.8(5.6)$ & 0.005 \\
\hline \multicolumn{4}{|c|}{ PDC threshold $\geq \mathbf{8 0} \%, \mathrm{n}(\%)$} \\
\hline SU class & $2,736(40.5)$ & $20(0.3)$ & $<0.001$ \\
\hline DPP-4 inhibitor class & $13(0.2)$ & $2,930(43.4)$ & $<0.001$ \\
\hline Antidiabetic drug (any) & $4,047(59.9)$ & $3,966(58.7)$ & 0.156 \\
\hline $\begin{array}{l}\text { Oral antidiabetic } \\
\text { drug (any) }\end{array}$ & $4,000(59.2)$ & $3,901(57.7)$ & 0.084 \\
\hline \multicolumn{4}{|c|}{ 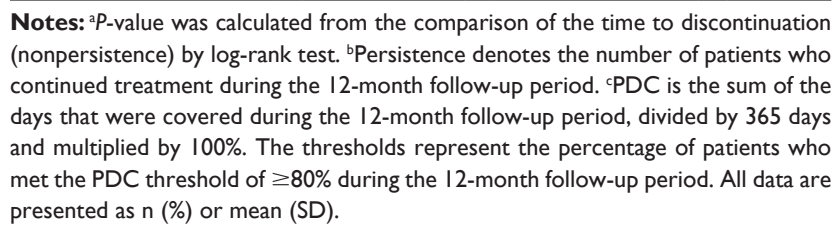 } \\
\hline \multicolumn{4}{|c|}{$\begin{array}{l}\text { Abbreviations: DPP-4, dipeptidyl peptidase-4; PDC, proportion of days covered; } \\
\text { SD, standard deviation; SU, sulfonylurea. }\end{array}$} \\
\hline
\end{tabular}

of the index drug compared to the SU cohort $(40.5 \%[2,736])$ (Table 2). Comparing the good adherence to OAD and overall antidiabetic drugs, a similar percentage of patients in the SU cohort met the PDC threshold of $\geq 80 \%$ compared to the DPP-4 inhibitor cohort (Table 2).

Table 3 provides a dynamic summary of the antidiabetic treatment regimens for up to 24 months for patients who discontinued their index drug. Of note, there was a high percentage of patients who did not initiate any antidiabetic medication following discontinuation of their index drug; a greater proportion of the SU cohort patients remained untreated. About $40 \%$ of patients resumed their index drug within 3 months of discontinuation. SU-treated patients were more likely to initiate metformin monotherapy, whereas DPP-4 inhibitor-treated patients were more likely to initiate combination OAD therapy after index drug discontinuation. Switching to the other combination therapy ( $\mathrm{SU}+$ metformin vs DPP-4 inhibitor + metformin) or moving to three-OAD combination therapy was infrequent. Patients in the SU cohort were more likely to initiate insulin therapy, whereas patients in the DPP-4 inhibitor cohort were more likely to initiate therapy with noninsulin injectables. However, the initiation of injectable therapy was minimal after index drug discontinuation for both cohorts. 


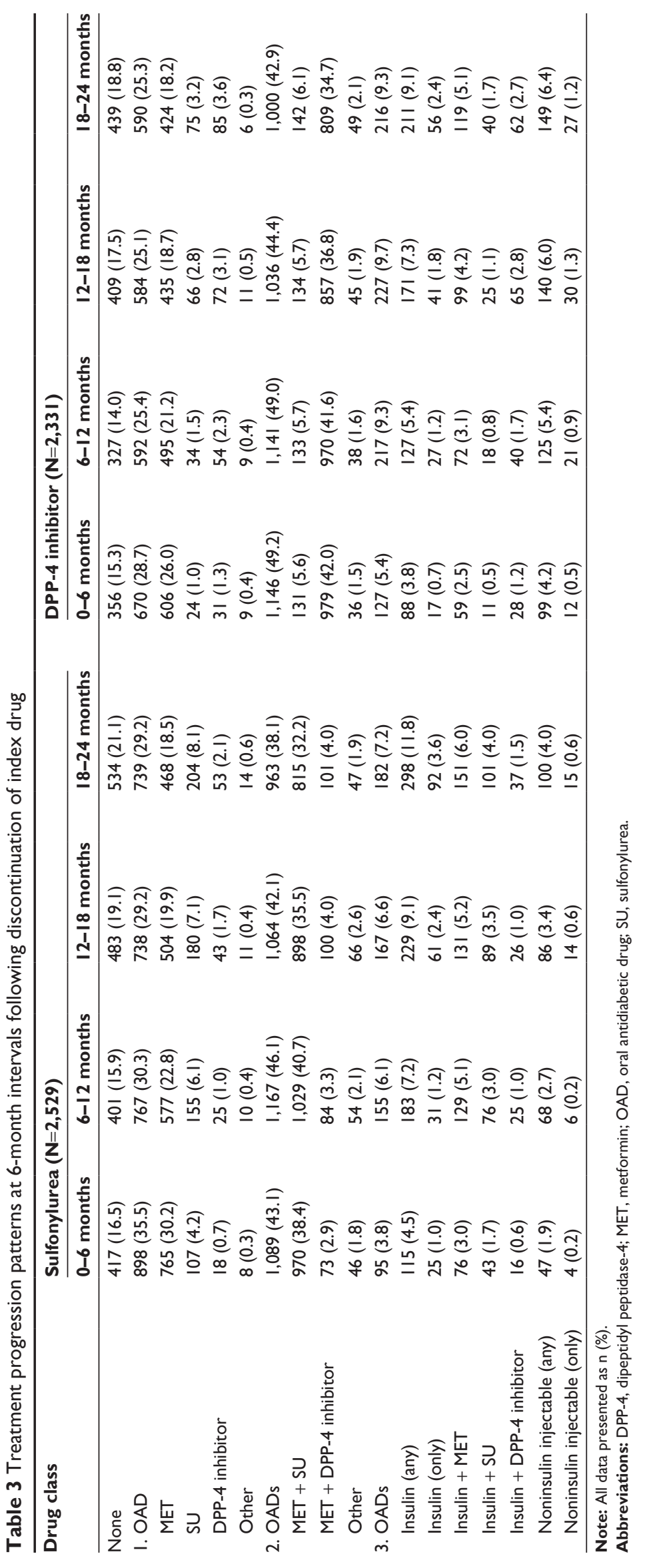




\section{Discussion}

The results of this retrospective study show slightly better adherence $(P<0.001)$ and persistence $(P<0.001)$ for DPP-4 inhibitor use than for SU. Although the differences observed were statistically significant, the differences may be too small to warrant clinical implications. Our results are similar to prior reports that support better adherence to and persistence with DPP-4 inhibitors than for SUs. ${ }^{10,11}$

The adherence results may be attributed to differences in the mechanisms of action of the drugs and the observed adverse side effects. The DPP-4 inhibitor agents are part of the incretin system medications, which increase the secretion of glucagon-like peptide-1 and gastric inhibitory peptide hormones by the small intestine. ${ }^{14}$ Agents in this class are able to mimic the "incretin effect", a greater insulin response after an oral glucose administration versus intravenous glucose administration. ${ }^{14}$ As suggested by Mishriky et al, ${ }^{15}$ DPP-4 inhibitors tend to be associated with less risk of hypoglycemia and weight gain in comparison to SUs. Considering this, patients in the DPP-4 inhibitor cohort may have experienced better treatment outcomes (less risk of hypoglycemia and minimal adverse events), resulting in better adherence compared to their SU counterparts. In addition, Doggrell and Warot ${ }^{16}$ suggest that better adherence is associated with better glycemic control (lower hemoglobin A1c [HbA1c or glycated $\mathrm{Hb}$ ] levels), but caution should be exercised while considering the impact of race/ethnicity and low income on glycemic control. Better adherence to OAD agents may also be achieved by improving treatment regimens to avoid/limit adverse side effects, prescribing agents with easier-to-use formulations and less dosing frequency, and use of better approaches by clinicians in treating individual patients. ${ }^{17}$

Our results also indicate that during the 24 months after discontinuation, as many as $40.7 \%$ and $42.0 \%$ of SU- and DPP-4 inhibitor-treated patients, respectively, resumed their index drug in combination with metformin. A general increase (SU:15.9\%-21.1\%; DPP-4 inhibitor: 14\%-18.8\%) in the percentage of patients not taking any agents was observed throughout the 24-month discontinuation follow-up period for both cohorts. Additionally, many patients did not switch to alternative OAD agents.

Another interesting finding of this study is that patients did not generally progress to use of more than two OADs, insulin, or noninsulin injectables in the 24 months after discontinuation. This may be attributed to the metformin background therapy for both cohorts. Rascati et al ${ }^{18}$ found that patients in the SU/thiazolidinedione cohort were $40 \%$ more likely to progress to insulin use compared to patients in the metformin/thiazolidinedione cohort. Their study also showed no significant difference between the SU and thiazolidinedione cohorts with metformin background therapy. Moreover, progression to noninsulin injectables was minimal, as also found in our study.

In sum, our results may be indicative of patient preferences for route of administration, dosing frequency, side effects, and other characteristics related to overall treatment regimen satisfaction with $\mathrm{OAD}$ use. Individual patients may have different needs and preferences, such as avoidance of weight gain, the risk of hypoglycemia, or medication costs, which play a role in their adherence to treatment regimens. More research is needed to understand the factors associated with medication selection, as well as adherence and persistence, and the reasons for which patients discontinue medications. Insufficient evidence exists to explore and evaluate 1) the high percentage of patients in this study who withdrew all antidiabetic medications and 2) the ways to improve medication adherence.

\section{Limitations}

In this study, HbA1c data were not available to evaluate the association between treatment outcomes and drug selection. Additional limitations are those common to most claims database studies. The claims database uses a standard set of medical care claims elements for items such as patient demographics and diagnosis/procedural codes, which may limit full patient profiling. Other limitations in the claims data include whether or not patients used medications as prescribed and reasons for discontinuation (financial hardship, no refill prescribed/available, etc).

\section{Conclusion}

This study evaluated adherence to and persistence with SUs and DPP-4 inhibitors in combination with metformin in propensity-matched cohorts. Additionally, treatment dispositions beyond discontinuation were captured over a 24-month period after discontinuation. While the study confirms differences in persistence and adherence among patients treated with the comparison drugs, it is noteworthy that there is a continuing and measurable gap in the knowledge about and research on adherence to both OAD and other treatments over longer periods.

\section{Acknowledgments}

This study was supported by Eli Lilly and Company. The authors wish to thank Ludi Fan for support with statistical analysis. 


\section{Disclosure}

XP, DL, OJV, and JPB are current employees of Eli Lilly and Company and own stock in the company. DJ is a former employee of Eli Lilly and Company. The authors report no other conflicts of interest in this work.

\section{References}

1. Inzucchi SE, Bergenstal RM, Buse JB, et al. Management of hyperglycemia in type 2 diabetes: a patient-centered approach: position statement of the American Diabetes Association (ADA) and the European Association for the Study of Diabetes (EASD). Diabetes Care. 2012; 35(6):1364-1379.

2. Inzucchi SE, Bergenstal RM, Buse JB, et al. Management of hyperglycemia in type 2 diabetes, 2015: a patient-centered approach: update to a position statement of the American Diabetes Association and the European Association for the Study of Diabetes. Diabetes Care. 2015;38(1):140-149.

3. Bailey CJ, Turner RC. Metformin. $N$ Engl J Med. 1996;334(9): 574-579.

4. Lamanna C, Monami M, Marchionni N, Mannucci E. Effect of metformin on cardiovascular events and mortality: a meta-analysis of randomized clinical trials. Diabetes Obes Metab. 2011;13(3):221-228.

5. Dezii CM, Kawabata H, Tran M. Effects of once-daily and twice-daily dosing on adherence with prescribed glipizide oral therapy for type 2 diabetes. South Med J. 2002;95(1):68-71.

6. Ingersoll KS, Cohen J. The impact of medication regimen factors on adherence to chronic treatment: a review of literature. J Behav Med. 2008;31(3):213-224.

7. Melikian C, White TJ, Vanderplas A, Dezii CM, Chang E. Adherence to oral antidiabetic therapy in a managed care organization: a comparison of monotherapy, combination therapy, and fixed-dose combination therapy. Clin Ther. 2002;24(3):460-467.

8. Rubin RR. Adherence to pharmacologic therapy in patients with type 2 diabetes mellitus. Am J Med. 2005;118(suppl 5A):27S-34S.
9. Zhang L, Zakharyan A, Stockl KM, Harada AS, Curtis BS, Solow BK. Mail-order pharmacy use and medication adherence among medicare Part D beneficiaries with diabetes. J Med Econ. 2011;14(5): 562-567.

10. Farr AM, Sheehan JJ, Curkendall SM, Smith DM, Johnston SS, Kalsekar I. Retrospective analysis of long-term adherence to and persistence with DPP-4 inhibitors in US adults with type 2 diabetes mellitus. Adv Ther. 2014;31(12):1287-1305.

11. Rathmann W, Kostev K, Gruenberger JB, Dworak M, Bader G, Giani G. Treatment persistence, hypoglycaemia and clinical outcomes in type 2 diabetes patients with dipeptidyl peptidase-4 inhibitors and sulphonylureas: a primary care database analysis. Diabetes Obes Metab. 2013;15(1):55-61.

12. Karve S, Cleves MA, Helm M, Hudson TJ, West DS, Martin BC. Good and poor adherence: optimal cut-point for adherence measures using administrative claims data. Curr Med Res Opin. 2009;25(9): 2303-2310.

13. D'Agostino RB Jr. Propensity score methods for bias reduction in the comparison of a treatment to a non-randomized control group. Stat Med. 1998;17(19):2265-2281.

14. Drucker DJ, Nauck MA. The incretin system: glucagon-like peptide-1 receptor agonists and dipeptidyl peptidase- 4 inhibitors in type 2 diabetes. Lancet. 2006;368(9548):1696-1705.

15. Mishriky BM, Cummings DM, Tanenberg RJ. The efficacy and safety of DPP4 inhibitors compared to sulfonylureas as add-on therapy to metformin in patients with Type 2 diabetes: a systematic review and meta-analysis. Diabetes Res Clin Pract. 2015;109(2):378-388.

16. Doggrell SA, Warot S. The association between the measurement of adherence to anti-diabetes medicine and the HbA1c. Int J Clin Pharm. 2014;36(3):488-497.

17. Nau DP. Recommendations for improving adherence to type 2 diabetes mellitus therapy - focus on optimizing oral and non-insulin therapies. Am J Manag Care. 2012;18(3 suppl):S49-S54.

18. Rascati KL, Richards KM, Lopez D, Cheng LI, Wilson JP. Progression to insulin for patients with diabetes mellitus using the Texas Medicaid database. Clin Ther. 2011;33(12):2016-2020.
Patient Preference and Adherence

\section{Publish your work in this journal}

Patient Preference and Adherence is an international, peer-reviewed, open access journal that focuses on the growing importance of patient preference and adherence throughout the therapeutic continuum. Patient satisfaction, acceptability, quality of life, compliance, persistence and their role in developing new therapeutic modalities and compounds to optimize

\section{Dovepress}

clinical outcomes for existing disease states are major areas of interest for the journal. This journal has been accepted for indexing on PubMed Central. The manuscript management system is completely online and includes a very quick and fair peer-review system, which is all easy to use. Visit http://www. dovepress.com/testimonials.php to read real quotes from published authors. 